\title{
Lusternik-Schnirelmann category of spaces with free fundamental group
}

\author{
JEFF STROM
}

\begin{abstract}
We find upper bounds for the Lusternik-Schnirelmann category of spaces with free fundamental groups. We prove, in particular, that if $X$ is a finite-dimensional CW complex with a free fundamental group, then $\operatorname{cat}(X) \leq \frac{2}{3} \operatorname{dim}(X)+1$. Our results specialize to resolve a question about the $\mathrm{L}-\mathrm{S}$ category of manifolds posed by Dranishnikov, Katz and Rudyak.
\end{abstract}

55M30; 55P99

\section{Introduction}

The Lusternik-Schnirelmann category $\operatorname{cat}(X)$ of a topological space $X$ is the least integer $n$ for which $X$ has an open cover $X=U_{0} \cup U_{1} \cup \cdots \cup U_{n}$ where each inclusion map $U_{k} \hookrightarrow X$ is nullhomotopic. Lusternik and Schnirelmann introduced this invariant in the 1930s for use in their study of critical points of smooth functions on manifolds. We refer to the book by Cornea, Lupton, Oprea and Tanré [1] for standard results on L-S category and to Whitehead [4] for background on homotopy theory.

In a recent paper [2], Dranishnikov, Katz and Rudyak proved that every compact manifold with cat $(M) \leq 2$ has a free fundamental group; this extends a well-known theorem about general spaces $X$ : if $\operatorname{cat}(X)=1$, then $\pi_{1}(X)$ must be a free group [1, $\mathrm{p}$ 44]. They then asked about the reverse implication: if $M$ is a compact manifold with free fundamental group, what can be said about the category of $M$ ? It is proved in [2] that the systolic category of a manifold $M$ with free fundamental group is bounded above by $\operatorname{dim}(M)-2$, prompting the authors to conjecture that $\operatorname{cat}(M) \leq \operatorname{dim}(M)-2$ (assuming $\operatorname{dim}(M) \geq 4$ ). The purpose of this note is to give a short proof of the following substantial generalization of their conjecture.

Theorem 1 If $X$ is a connected $C W$ complex whose fundamental group is free and whose universal cover $\tilde{X}$ is $(n-1)$-connected, then

$$
\operatorname{cat}(X) \leq\left\lfloor\frac{\operatorname{dim}(X)}{n+1}\right\rfloor+\left\lceil\frac{\operatorname{dim}(X)}{n+1}\right\rceil .
$$


We obtain our answer to the question of Dranishnikov, Katz and Rudyak as an immediate corollary. It is unfortunate, but true, that we are unable to resolve the case $\operatorname{dim}(X)=4$.

Corollary 2 If $X$ is a connected $C W$ complex with free fundamental group and $\operatorname{dim}(X)>4$, then $\operatorname{cat}(X) \leq \operatorname{dim}(X)-2$.

Since the universal cover of a path connected space is 1-connected by definition, we have the following consequence, which is slightly weaker - but easier to appreciate than the $n=2$ case of Theorem 1 .

Corollary 3 If $X$ is any connected $C W$ complex with free fundamental group, then

$$
\operatorname{cat}(X) \leq \frac{2}{3} \operatorname{dim}(X)+1 \text {. }
$$

This corollary refines the well-known result that $\operatorname{cat}(X) \leq \operatorname{dim}(X)$ for any space $X$ [1, Theorem 1.7].

\section{Categorical sequences}

The key to the proof of Theorem 1 is the formalism of the categorical sequence of a space, which was introduced by the author in [3]. Before defining it, we must clarify our use of the term "an $n$-skeleton" for a (path connected) space $X$. Every space $X$ has a CW approximation, which is a weak homotopy equivalence $\bar{X} \rightarrow X$ where $\bar{X}$ is a CW complex. If $X_{n}$ is an $n$-dimensional CW complex, and $X_{n} \rightarrow X$ is a map such that the induced map $\pi_{k}\left(X_{n}\right) \rightarrow \pi_{k}(X)$ is an isomorphism for $k<n$ and a surjection for $k=n$, then one can attach to $X_{n}$ cells of dimension $n+1$ and greater to obtain a CW approximation of $X$ extending the given map. For this reason, we call any map $X_{n} \rightarrow X$ as above an $n$-skeleton for $X$. We denote by cat $X_{X}\left(X_{n}\right)$ the category of $X_{n}$ in $X$; ie, the least integer $k$ for which $X_{n}=U_{0} \cup \cdots \cup U_{k}$ and each composition $U_{i} \hookrightarrow X_{n} \rightarrow X$ is nullhomotopic. It was proved in [3] that the number cat $_{X}\left(X_{n}\right)$ depends only on the homotopy type of $X$ and the dimension $n$, and not on the choice of skeleton $X_{n} \rightarrow X$. Note that with this terminology, one always refers to "an $n$-skeleton" of $X$ and never to "the $n$-skeleton" of $X$.

If $X$ is a path connected space, then its categorical sequence is the function $\sigma_{X}: \mathbb{N} \rightarrow$ $\mathbb{N} \cup\{\infty\}$ given by

$$
\sigma_{X}(k)=\inf \left\{n \mid \operatorname{cat}_{X}\left(X_{n}\right) \geq k\right\}
$$

with the usual convention that $\inf (\varnothing)=\infty$. If $X$ is a finite dimensional CW complex, then $\operatorname{cat}(X)$ is the largest value of $k$ for which $\sigma_{X}(k) \leq \operatorname{dim}(X)$. The key to calculations with categorical sequences is their superadditivity property: for all $k$ and $l$, $\sigma_{X}(k+l) \geq \sigma_{X}(k)+\sigma_{X}(l)[3$, Theorem 18]. 
Before proving Theorem 1, we establish a restriction on the categorical sequences of spaces with free fundamental groups.

Proposition 4 Let $X$ be a connected CW complex. Then $\pi_{1}(X)$ is free if and only if $\sigma_{X}(2)>2$.

Proof If $\sigma_{X}(2)>2$, then by [3, Proposition 9], cat $\left(X_{2}\right)=$ cat $_{X}\left(X_{2}\right)=1$. It follows that $\pi_{1}(X) \cong \pi_{1}\left(X_{2}\right)$ is a free group.

For the converse, suppose that $\pi_{1}(X)$ is free on the set $\mathcal{G}=\left\{\gamma_{i} \mid i \in I\right\}$. Set $V_{1}=$ $\bigvee_{i \in I} S^{1}$ and define $c_{1}: V_{1} \rightarrow X$ to be $\gamma_{i}$ on the $i$-th summand. Since $c_{1}$ induces an isomorphism on $\pi_{1}$, it can be used as a 1 -skeleton for $X$. To form $X_{2}$, set $V_{2}=\bigvee_{j} S^{2}$ and define $c_{2}: V_{2} \rightarrow X$ so that $\left(c_{2}\right)_{*}: \pi_{2}\left(V_{2}\right) \rightarrow \pi_{2}(X)$ is surjective. Then $\left(c_{1}, c_{2}\right): V_{1} \vee V_{2} \rightarrow X$ is a $2-$ skeleton of $X$. Since cat $\left(V_{1} \vee V_{2}\right)=1$, we have $\sigma_{X}(2)>2$.

\section{Proof of Theorem 1}

First we construct a convenient $\mathrm{CW}$ decomposition for $X$, continuing with the notation from the proof of Proposition 4. Since $\pi_{k}(X)=0$ for $1<k<n$, the map $c_{1}: V_{1} \rightarrow X$ is an isomorphism on $\pi_{k}$ for $k<n$, and we may take $X_{n-1}=V_{1}$. Then, just as in the earlier proof, we may take $X_{n}=V_{1} \vee V_{n}$ where $V_{n}=\bigvee S^{n}$. Now $\operatorname{cat}\left(X_{n}\right)=1$ and so $\sigma_{X}(2) \geq n+1$. It follows that

$$
\sigma_{X}(2 q) \geq q \cdot \sigma_{X}(2)=q(n+1) \quad \text { and } \quad \sigma_{X}(2 q+1) \geq q(n+1)+1 .
$$

Let $\operatorname{dim}(X)=d<\infty$; then $\operatorname{cat}(X)$ is equal to the largest value of $k$ for which $\sigma_{X}(k) \leq d$. Writing $d=q(n+1)+r$ with $0 \leq r<n+1$, we have

$$
\begin{aligned}
k & \leq \begin{cases}2 q & \text { if } r=0 \\
2 q+1 & \text { if } r>0\end{cases} \\
& \leq\left\lfloor\frac{d}{m}\right\rfloor+\left\lceil\frac{d}{m}\right\rceil,
\end{aligned}
$$

proving the theorem.

\section{Concluding remarks}

(1) I received helpful advice from Martin Arkowitz, Daniel Tanré, John Oprea and Mikhail Katz while preparing this paper.

Algebraic 83 Geometric Topology, Volume 7 (2007) 
(2) The superadditivity condition satisfied by categorical sequences is a substantial restriction on the possible sequences; the result of this paper shows that this restriction can lead to interesting results. Therefore we ask: which superadditive sequences are the categorical sequences of spaces? More specifically, is there a space $X$ with categorical sequence $\sigma_{X}=(0,1,3,4, \ldots)$ ? A negative answer would resolve the remaining case of our motivating question.

(3) In [3, Lemma 1.3], it is proved that if $X$ is a simply connected space such that $H^{n}(X ; A)=0$ for all coefficient groups $A$, then $X$ has an $(n-1)$-dimensional $n$-skeleton. The proof given there makes use of an unusual set theoretic hypothesis which guarantees a positive solution to Whitehead's problem. We thank Nick Kuhn for pointing out that there is a simple proof which does not require any oppressive hypotheses: using the Universal Coefficient isomorphism, we find that $\operatorname{Ext}\left(H_{n-1}(X ; \mathbb{Z}), A\right)=0$ for all $A$, so $\operatorname{Hom}\left(H_{n-1}(X ; \mathbb{Z}),-\right)$ is an exact functor; this implies $H_{n-1}(X ; \mathbb{Z})$ is a projective $\mathbb{Z}$-module and hence free. The rest of the proof given in [3] works as written.

\section{References}

[1] O Cornea, G Lupton, J Oprea, D Tanré, Lusternik-Schnirelmann category, Mathematical Surveys and Monographs 103, American Mathematical Society, Providence, RI (2003) MR1990857

[2] A N Dranishnikov, M G Katz, Y B Rudyak, Small values of Lusternik-Schnirelmann and systolic categories for manifolds arXiv:0706.1625v2

[3] R Nendorf, N Scoville, J Strom, Categorical sequences, Algebr. Geom. Topol. 6 (2006) 809-838 MR2240916

[4] G W Whitehead, Elements of homotopy theory, Graduate Texts in Mathematics 61, Springer, New York (1978) MR516508

Department of Mathematics, Western Michigan University

1903 W. Michigan Ave., Kalamazoo, MI 49008

jeff.strom@wmich.edu

http://homepages.wmich.edu/ jstrom/

Received: 11 July 2007 Revised: 2 October 2007 